\title{
Les lettres de Vincent Voiture: du discours familier au texte littéraire
}

\section{Sophie Rollin}

\section{(2) OpenEdition}

1 Journals

\section{Édition électronique}

URL : http://journals.openedition.org/studifrancesi/7789

DOI : ERREUR PDO dans /localdata/www-bin/Core/Core/Db/Db.class.php L.34 : SQLSTATE[HY000]

[2006] MySQL server has gone away

ISSN : 2427-5856

\section{Éditeur}

Rosenberg \& Sellier

Édition imprimée

Date de publication : 1 juillet 2009

Pagination : 251-263

ISSN : 0039-2944

\section{Référence électronique}

Sophie Rollin, «Les lettres de Vincent Voiture: du discours familier au texte littéraire », Studi Francesi [En ligne], 158 (LIII | II) | 2009, mis en ligne le 30 novembre 2015, consulté le 07 janvier 2021. URL : http://journals.openedition.org/studifrancesi/7789; DOI : https://doi.org/ERREUR PDO dans / localdata/www-bin/Core/Core/Db/Db.class.php L.34 : SQLSTATE[HY000] [2006] MySQL server has gone away

\section{(c) (i) $\odot$}

Studi Francesi è distribuita con Licenza Creative Commons Attribuzione - Non commerciale - Non opere derivate 4.0 Internazionale. 


\section{Les lettres de Vincent Voiture: du discours familier au texte littéraire}

Madame, voici sans doute la plus belle aventure que Roland ait jamais eue, et lorsqu'il défendait seul la couronne de Charlemagne, et qu'il arrachait les sceptres des mains des rois, il ne faisait rien de si glorieux pour lui qu'à cette heure qu'il a l'honneur de baiser les vôtres. Le titre de furieux, sous lequel il a couru jusqu'ici toute la terre, ne doit pas empêcher que vous ne lui accordiez cette grâce, ni vous faire craindre sa rencontre ${ }^{1}$.

Ainsi commence la plus ancienne lettre de Vincent Voiture qui soit parvenue jusqu'à nous. Il s'agit d'une lettre authentique et privée, adressée aux alentours de $1625^{2}$ à la femme qu'il courtisait alors, Mme de Saintot ${ }^{3}$, pour accompagner le présent d'un exemplaire du Roland furieux, dans la récente traduction de Rosset ${ }^{4}$. Mais une lettre particulièrement originale, qui se distingue par un usage subtil du pastiche. Dès la première phrase, en déclarant que Roland a «l'bonneur de baiser les vôtres [(vos) mains]», Voiture confond habilement l'ouvrage et son personnage éponyme pour transfigurer l'acte d'ouvrir le livre en geste d'hommage amoureux. Jusqu'à la fin de la lettre, il imite l'univers et le style du Roland furieux en utilisant l'ouvrage de l'Arioste comme un intertexte chargé d'exprimer la signification amoureuse de son propre discours. La galanterie ${ }^{5}$ séduit la dame. Mais aussi tout le milieu mondain de l'époque: la lettre est lue dans les salons et circule sous forme de copies manuscrites. Voiture, qui jusqu'alors s'était efforcé en vain d'entrer dans la clientèle d'un grand seigneur, est présenté à la marquise de Rambouillet et nommé, quelques mois plus tard, introducteur des ambassadeurs à l'Hôtel d'Orléans. Sa fortune est faite et son destin scellé: il demeurera au service de Monsieur et fréquentera les salons les plus distingués.

Aussi rédige-t-il, au cours de sa vie, un certain nombre de lettres dans le cadre de ses fonctions diplomatiques ou pour maintenir le contact avec la société parisienne quand il est en mission, et il compose des poésies courtes et enjouées qui égayent les réunions mondaines. Des écrits ressortissant donc à ses fonctions professionnelles ou aux usages de la sociabilité, qu'il ne publiera jamais. A sa mort, cependant, en 1648, un de ses neveux, Martin Pinchesne, entreprend de les rassembler et les publie, deux ans plus tard, en 1650, sous le titre: Euvres de M. de Voiture ${ }^{6}$, présentant ainsi

(1) Vincent Voiture, lettre à Mme de Saintot, antérieure à 1625, dans Euvres, lettres et poésies, éd. A. UBICINI [1855] reprint Genève, Slatkine, 1967, 2 voll., t. I, pp. 17-18.

(2) La date de la lettre est incertaine. Elle est probablement un peu antérieure à 1625 .

(3) Marguerite Vion, fille de Nicolas Vion d'Oinville, auditeur des comptes normands, et sœur du poète Charles Vion d'Alibray. Elle épouse en 1622 Pierre de Saintot, trésorier de France à Tours.

(4) Traduction du Roland furieux par François DE Rosset publiée en 1615 (Paris, R. Fouet, réédi- tée en 1624 et 1643 ).

(5) J'emploie le mot «galanterie» dans une de ses acceptions au XVII ${ }^{e}$ siècle: Richelet consigne le sens de «compliment»: «Fleuréttes, douceurs amoureuses, manière civile Es agréable de dire, ou de faire les choses», et celui de «ouvrage exprimant des compliments»: «Ouvrage galant, plein d'esprit et d'amour, en vers ou en prose». Dictionnaire françois contenant les mots et les choses [1680], article «Galanterie».

(6) Euvres de M. de Voiture, Paris, Augustin Courbé, 1650. 
l'ouvrage comme une somme littéraire. Le livre est pourtant composé aux trois quarts de lettres privées et familières ${ }^{7}$, complétées par une centaine de courtes poésies. Il connaît un immense succès: aussitôt épuisé, une seconde édition augmentée voit le jour avant la fin de la même année. A cette reconnaissance par le public s'ajoute celle des gens de Lettres: La Fontaine revendiquera Voiture comme «un maître», Mme de Sévigné et La Bruyère le présenteront comme un «modèle» incarnant, selon le Père Bouhours, l'esprit français ${ }^{8}$.

Le décalage entre la manière dont Voiture semble avoir pratiqué l'écriture épistolaire de son vivant et ce qu'il advint de ses écrits après sa mort souligne l'ambivalence de ses lettres: en tant que lettres authentiques, échangées dans une sphère privée et rédigées dans un registre moyen, elles prennent la forme du discours familier, mais leur publication leur accorde un statut de texte littéraire et la réception du recueil consacre Voiture comme un écrivain.

Ces lettres posent ainsi la question de la littérarité du discours épistolaire et des modalités selon lesquelles s'est formée la conception d'un «genre épistolaire» au cours du XVII ${ }^{\mathrm{e}}$ siècle. Elles invitent à examiner si, en dehors de l'acte de la publication qui leur offre un statut de texte littéraire, on peut repérer des indices de littérarité dans le discours lui-même, et quelle forme ils prennent. S'agissant de lettres, on partira des éléments objectifs que présentent leurs conditions de diffusion, on observera ensuite comment le discours se situe par rapport à une tradition épistolaire établie, et on s'interrogera enfin sur les conséquences qu'une écriture passant par l'imitation peut avoir sur le statut du discours.

$* * *$

\section{Diffusion et réception des lettres de Voiture}

Le succès immédiat des lettres de Voiture tient en partie au fait qu'elles n'étaient pas entièrement inconnues. Elles étaient adressées à un destinataire précis et acheminées par la poste, mais beaucoup étaient ensuite lues en public. Par exemple, la lettre à Mme de Saintot citée au début de cet article «a tant couru», dit Tallemant des Réaux ${ }^{9}$, qu'elle a immédiatement fait connaître Voiture dans le milieu mondain. Ce mode de diffusion auprès d'un public élargi s'inscrit dans les modalités d'écriture du discours car sa situation d'énonciation tend à dépasser les limites du duo habituelle-

(7) Tout ce qui faisait directement référence aux réalités du temps, comme les écrits politiques ou le nom des femmes auxquelles sont adressées les lettres amoureuses en a été retranché. TALLEMANT DES RÉAuX déclare que «Martin [Pinchesne] a sottement effacé des noms, en y mettant des estoiles, au lieu de les garder pour les remettre quelque jour». Il proteste également contre «M. Chapelain et $\mathrm{M}$. Conrart, qui ont tant estoilé ce pauvre livre», Historiettes, «Voiture», éd. A. ADAM, Paris, Gallimard, 1960 («Bibliothèque de la Pléiade»), t. I, p. 499.

(8) La FOnTAINE: «J'ai profité dans VoiturelEt Marot par sa lecturelM'a fort aidé, j'en conviens [...] J'oubliais maittre François, dont je me dis encore le disciple, aussi bien, que celui à maître Vincent, et celui de maitre Clément. Voilà bien des maîtres pour un écolier de mon âge», lettre à M. de Saint-Evremond du 8 décembre 1687, dans Euvres complètes, éd. J. Marmier, Paris, Le Seuil,
1965, «L'Intégrale», p. 41. MME DE SÉVIGNÉ déclare à Bussy-Rabutin, à propos de Voiture: «Vous me faites rire quand vous croyez que quelqu'un puisse écrire comme lui», dans Correspondance, éd. R. DuCHÊNE, Paris, Gallimard («Bibliothèque de la Pléiade»), III, 1209, p. 883. LA BRUYÈRE: «Voiture toujours original, soit qu'il invente, soit qu'il traduise; qui a été au-delà de ses modèles, modèle lui-même difficile à imiter», Discours de réception à l'Académie, 15 juin 1693, dans Les Caractères, éd. E. BuRY, Paris, Le livre de Poche, 1995, p. 626. Père DomiNIQUE BOUHOURS: «On peut dire que Voiture nous a appris cette manière d'écrire aisée et délicate qui règne présentement. Avant lui, on pensait n'avoir de l'esprit que quand on parlait Balzac tout pur et qu'on exprimait de grandes pensées avec de grands mots», Entretiens d'Ariste et d'Eugène, IV, éd. F. Brunot, Paris, Armand Colin, 1962, p. 133.

(9) Tallemant des RÉaux, op. cit., t. I., p. 491. 
ment formé par les correspondants. Une lettre à Mlle Paulet, par exemple, projette l'image d'un destinataire multiple en ajoutant des messages à l'intention de Mme de Rambouillet, de Mme de Clermont, de Mlle de Rambouillet, du Marquis de Pisany et du cardinal de La Valette:

[...] Mais une des plus grandes marques que je pusse donner que l'air d'Afrique m'a inspiré quelque félonie, c'est que j'ai écrit déjà trois pages, et que j'ai pensé achever cette lettre sans parler de Madame de Rambouillet $t^{10}$. Je vous assure pourtant qu'en quelque part que je sois, elle est toujours dans mon cœur et dans mon souvenir, et même en ce moment, ben che $d u$ tanta lontananza, li fo umilissima riverenza, et suis son très humble et très obéissant serviteur, Brambano. Tant que je serai hors de la chrétienté, je n'oserais rien dire à Madame de Clermont pour Mademoiselle de Rambouillet. Je crois qu'elle ne me voudra pas plus de mal pour cela. J'espère lui payer quelque jour le plaisir que j'ai eu d'ouïr les aventures d'Alcidalis ${ }^{11}$, en lui racontant les miennes. Je lui ferai entendre des choses étranges et incroyables et, pour ses fables, je lui rendrai des histoires. Votre serviteur ${ }^{12}$ a toujours dans mon esprit la place que son mérite, et l'affection qu'il me fait l'honneur d'avoir pour moi lui doivent donner. Il y a un de vos amis $^{13}$, Mademoiselle, que j'aime avec tant de passion, que j'en oublie mon devoir, et qu'il ne me souvient pas de dire combien je le respecte, et je l'honore. [...]

Les propos adressés au cardinal sont si longuement développés dans la suite de la lettre qu'ils constituent, in fine, une seconde lettre enchâssée dans la première ${ }^{14}$. Les lettres de Voiture débordent ainsi le cadre traditionnel du discours épistolaire et prennent la forme de lettres «semi-privées».

Les personnes mentionnées dans la lettre à Mlle Paulet redessinent le cercle de l'Hôtel de Rambouillet dont Voiture était l'un des principaux habitués et où ses lettres étaient lues en priorité. La «Chambre bleue d'Arthénice» ${ }^{15}$, étant à la fois l'un des premiers «salons», pour employer une appellation moderne, et le plus prestigieux, est fréquentée par la partie la plus cultivée de l'aristocratie et par les gens de Lettres de l'époque. A une époque où c'est le jugement des femmes qui fixe les critères du bon usage et du goût, où les «salons» sont parfois nommés des «académies» et constituent le versant mondain de la critique artistique et littéraire, le cercle de la marquise de Rambouillet intervient comme une suprême instance d'évaluation dans le domaine des Lettres et des $\operatorname{arts}^{16}$. Non seulement il sert ainsi de «médiateur» ${ }^{17}$ privilégié entre Voiture et le lectorat mondain, diffusant ses écrits sans qu'ils passent par une publication effective, mais de plus il en assure la reconnaissance et la promotion. Dans ces conditions, l'ancrage social de Voiture à l'Hôtel de Rambouillet l'inscrit concrètement et symboliquement dans le champ littéraire tel qu'il est constitué à cette époque.

C'est pourquoi la publication de ses écrits était attendue de longue date. Vaugelas en témoigne qui, après avoir loué le style d'un épistolier en qui on peut reconnaître Voiture, en appelle au public pour l'inciter à rendre ses lettres publiques:

(10) Nous soulignons ce destinataire second ainsi que les suivants.

(11) Roman que Voiture et Mademoiselle de Rambouillet avaient commencé à écrire ensemble.

(12) Le marquis de Pisany.

(13) Le cardinal de La Valette.

(14) Voir lettre à Mlle Paulet, Ceuta, 7 août 1633, op. cit., t. I, pp. 161-163.

(15) L'Hôtel de Rambouillet. «Arthénice» est une anagramme composée par Malherbe pour désigner la marquise de Rambouillet.

(16) C'est ce que déclare AnToine Furetière dans sa Nouvelle allégorique ou Histoire des der- niers troubles arrivés au Royaume d'Eloquence [1658], éd. M. BombarT et N. Schapira, Toulouse, Société de littératures classiques-Paris, Champion, 2004.

(17) J'emprunte ce terme à Alain VIALA qui l'emploie dans son analyse du rôle joué par les salons dans la constitution du champ littéraire au XVII ${ }^{\mathrm{s}}$ siècle. Naissance de l'écrivain. Sociologie de la littérature à l'âge classique, «Le sens commun», éd. de Minuit, Paris, 1985. A propos des salons comme «lieux de médiation», voir notamment pp. 132137 et à propos de l'influence de l'Hôtel de Rambouillet, voir pp. 154-155. 
En cette sorte de lettres [galantes], la France peut se vanter d'avoir une personne à qui tout le monde cède. Athènes même ni Rome si vous en ôtez Cicéron, n'ont pas de quoi le lui disputer, et je le puis dire hardiment, puisqu'à peine parait-il qu'un genre d'écrire si délicat, leur ait été seulement connu. Aussi tous les goûts les plus exquis font leurs délices de ses lettres, aussi bien que de ses vers, et de sa conversation, où l'on ne trouve pas moins de charmes. Je tiendrais le public bien fondé à intenter une action contre lui pour lui faire imprimer ses œuvres ${ }^{18}$.

Il fait valoir une sorte de «droit du lecteur» à accéder à un patrimoine qui lui reviendrait et indique, par là, que les lettres de Voiture étaient regardées comme des textes littéraires par ses contemporains avant même d'entrer dans le domaine public.

Toutefois, un décalage apparaît entre d'une part, leur diffusion et leur réception, et d'autre part l'attitude de l'épistolier qui semble, apparemment, inscrire ses productions dans les marges de la littérature. Non seulement il n'entreprendra jamais cette édition qui lui est réclamée, préférant laisser circuler ses écrits dans des cercles privilégiés, mais de plus, il pratique une forme d'écriture qui, jusqu'alors, n'était pas reconnue comme une forme littéraire.

\section{Des discours familiers?}

En effet, à l'époque où les CEuvres de Voiture paraissent, l'épistolaire n'est pas regardé comme un genre littéraire. Les lettres latines, authentiques ou fictives, rédigées par les grands auteurs antiques, constituent des modèles de référence. Mais très peu de lettres en français ont fait l'objet d'une publication. Au siècle précédent, Etienne Pasquier a ajouté à ses poésies des lettres d'amour authentiques dans un recueil intitulé Rimes et prose (1552). Un siècle, à quelques années près, sépare cet ouvrage du premier recueil de lettres publié par Guez de Balzac, en $1624^{19}$, qui rassemble des lettres éloquentes développant des sujets d'ordre philosophique, politique ou moral. A cette époque, deux formes de lettres sont donc publiées, et ainsi reconnues comme des textes littéraires: la lettre d'amour, sur le modèle des Héroïdes ${ }^{20}$ d'Ovide, et la lettre savante, sur le modèle des lettres de Cicéron ou de Sénèque.

Ces deux modèles apparaissent dans l'édition des Euvres de Voiture qui comporte 55 lettres d'amour, ainsi que 16 lettres à Pierre Costar traitant de questions savantes. Mais reste l'essentiel: 147 lettres familières, adressées à des correspondants divers, soit $67 \%$ du recueil ${ }^{21}$. De plus, dans ses lettres d'amour et ses lettres érudites, Voiture se démarque ostensiblement des modèles épistolaires ayant fait leur entrée dans le domaine public.

Dans les lettres à ses amantes, il s'inspire des Héroïdes et de la poésie pétrarquiste du XVI ${ }^{\mathrm{e}}$ siècle, mais substitue au ton élégiaque de ces textes une tonalité plaisante. Par exemple, dans une série de dix lettres adressées à une dame qui vient de partir

(18) VAUGELAS, Remarques sur la langue française [1647], éd. J. STREICHER, Genève, Slatkine Reprints, 1970 , p. 478.

(19) Alain Viala a analysé les grandes traditions épistolaires sur lesquelles se fonde l'usage de la lettre au XVII ${ }^{\mathrm{e}}$ siècle dans La genèse des formes épistolaires en français et leurs sources latines et européennes. Essai de chronologie distinctive (XVI'-XVII ${ }^{e}$ s.), «Revue de littérature comparée», avril-juin 1981, 218 , pp. 168-183. Voir notamment pp. 171-172, à propos d'Etienne Pasquier, et p. 173, à propos de

\section{Guez de Balzac.}

(20) Marie-Claire Chatelain souligne le succès des Héroïdes au XVII ${ }^{\mathrm{e}}$ siècle, notamment dans la première moitié du siècle dans son ouvrage sur la réception et l'influence d'Ovide au XVII ${ }^{\mathrm{e}}$ siècle: Ovide savant, Ovide galant. Ovide en France dans la seconde moitié du XVII siècle, Paris, Champion, «Lumière classique», 2008, voir notamment p. 21.

(21) L'ouvrage comporte également deux lettres «en vieux langage» et une lettre écrite en espagnol, présentées dans une section à part. 
pour un séjour prolongé22, il développe le topos de la mort par amour en employant le lexique de la mort au sens littéral et non figuré. Dès le début de la série, il mine la rhétorique qu'il emploie en signalant ironiquement le paradoxe d'une lettre qui exprime l'intensité du sentiment par l'image de la mort tout en attestant que l'épistolier est en vie:

Quand je songe que demain vous ne serez plus ici, je trouve étrange qu'aujourd'hui je sois en vie, et je suis prêt d'avouer avec vous qu'il y a de l'affectation en cet amour que je fais paroître, quand je pense que je respire encore, et que ce déplaisir n'achève pas de me tuer ${ }^{23}$.

D’une lettre à l'autre, l'humour est de plus en plus marqué et la série s'achève sur des raisonnements par l'absurde:

[...] sans mentir, il seroit impossible que je pusse tant vivre si je n'espérois pas bientôt d'en mourir. [...] Néanmoins, [...] dites-moi si ma folie pouvoit avoir une fin plus heureuse que celle-là, et ce qu'il fût arrivé de moi, si j'eusse vécu davantage ${ }^{24}$.

Tandis que, dans des lettres telles que les Héroïdes, la tonalité élégiaque inscrit l'expression du sentiment amoureux dans un registre noble qui souligne la littérarité du discours, dans celles de Voiture, la tonalité plaisante, contrastant avec le traitement traditionnel de la thématique amoureuse, leur donne l'apparence d'un discours familier sans vocation littéraire.

Le même paradoxe apparaît dans les lettres adressées à Costar: Voiture y affiche une certaine désinvolture à l'égard des sujets savants alors que ces échanges constituent une véritable correspondance érudite, nourrie de citations latines, traitant des sujets littéraires ou philologiques. Dès la première lettre de cette série ${ }^{25}$, il adopte le style de la conversation familière en mêlant des questions d'ordre général et des préoccupations présentes, et commence son discours en feignant de s'arracher à regret à ses préoccupations amoureuses pour accorder de son temps à l'entretien de Costar:

Au lieu de me parler du sujet de mon déplaisir, et de me dire ce que vous en jugez (car il y a lieu d'exercer des conjectures là-dessus, aussi bien que sur le plus obscur passage de Tacite), vous m'alléguez Lampridus et Athénée, quam inepte! et en un temps où je dispute de moimême savoir si Mme de ${ }^{* * * *}$ m'aime, ou si elle ne m'aime pas, et que cela est devenu une chose problématique, vous me venez m'entretenir de Pharaon ${ }^{26}$.

Il se démarque donc du modèle traditionnel de la lettre savante en faisant s'entrechoquer registre et thématique: l'allusion à $M m e$ de $e^{\text {**⿰冫欠 }}$ vient rompre le registre savant des références à Tacite, Lampridus, Athénée et de la citation latine «quam inepte! $\gg^{27}$. Il dégrade même l'image docte de son correspondant en ne sollicitant

(22) Lettres $n^{\circ} 9$ à $n^{\circ} 18$, op. cit., t. II, pp.184199. 185.

(23) Lettre $\mathrm{n}^{\circ} 15$ à Mme $\mathrm{M}^{* * *}$, ibid., t. II, pp. 184-

(24) Lettre $n^{\circ} 15$ à Mme स** $^{* *}$ ibid., t. II, p. 192194. Pour une analyse plus détaillée de ces lettres, voir mon ouvrage Le Style de Vincent Voiture: une esthétique galante, Saint-Étienne, Publications de l'Université de Saint-Étienne, 2006, p. 143-147.

(25) Bien que certaines lettres de Voiture soient perdues, celles qui évoquent les affaires politiques, par exemple, il est fort possible que cette lettre soit effectivement celle qui débute sa correspondance avec Costar puisque ce dernier, qui publiera par la suite leurs Entretiens, semble avoir conservé une copie de toutes les lettres qu'ils échangeaient.

(26) Lettre à Pierre Costar, entre 1638 et 1642 , op. cit., t. II, pp. 83-84.

(27) Cette citation est identifiée comme une référence à une lettre de Sénèque à Lucilius par CÉCILE TARDY, qui prépare une nouvelle édition des Entretiens de Voiture et de Costar (Mémoire de thèse soutenue à l'Université de Toulouse le 29 novembre 2008, vol. 2, p. 44, note 138). Toutefois, remise dans le contexte de cette lettre de Sénèque, la citation entretient des rapports assez lointains avec le propos de Voiture: «Illud deinde mecum loqui coepi, quam inepte quaedam magis aut minus ti- 
pas son avis sur une question relevant de ses compétences, mais sur une affaire de cœur déclarée aussi «problématique» que «le plus obscur passage de Tacite». Mais, passée cette entrée en matière décalée et facétieuse, il confesse de bon gré l'intérêt qu'il prend aux sujets développés par Costar: «Néanmoins, je vous avoue que je n'ai point été fâché de lire tout ce que vous $m^{\prime}$ écrivez» ${ }^{28}$, ajoute-t-il. Ces effets de décalage et de rupture mettent donc en évidence un effort de trompe-l'œil proprement littéraire. Dès lors, ces lettres témoignent d'une érudition d'autant plus solide qu'elle semble spontanée, et l'apparente facilité avec laquelle elle s'exprime y ajoute une grande virtuosité littéraire.

Cette littérarité en trompe-l'œil caractérise plus manifestement encore les lettres familières de Voiture qui sont ancrées sur le présent. La feinte désinvolture qu'il affiche s'étend à tout ce qui relève du domaine «sérieux». Une lettre adressée à Claude de Chaudebonne, fidèle du duc d'Orléans ${ }^{29}$, fait pendant à la lettre à Costar que nous venons de mentionner. Après avoir séjourné un an à Madrid, assurant la liaison entre le duc d'Orléans et l'Espagne, en pleine guerre de Trente Ans, et pensionné par le premier ministre espagnol ${ }^{30}$, Voiture se prétend davantage occupé par les femmes que par les affaires politiques:

Etant à la veille de mon partement, je vous écris avec le peu de loisir que vous pouvez imaginer que doit avoir un homme aussi négligent que moi et qui a accoutumé de remettre toutes choses jusqu'au dernier jour. Outre quelques affaires qui me restent, il me faut aller dire adieu à dona Antonia, à dona Inez et Isabelina, à la Guzmana, à la Catalana, y las dos Toledanas. Il faut que j'envoie un recade à dona Elvira; que j'écrive un billet à dona Urraza, et que je donne des chapin y un manto à dona Alonza, et un chapelet à sa mère dona Pedraza ${ }^{31}$.

Voiture se dit «négligent», illustrant cette attitude par un discours qui escamote le politique pour s'attarder sur les plaisirs de l'amour, et dresse de lui-même le portrait d'un Dom Juan frivole. Dans cette lettre qui ne ressortit pas à un modèle épistolaire particulier, il enfreint le principe de la convenance censé régler tous les échanges, par un discours en décalage avec la situation, avec ses propres fonctions, et enfin avec la

meremus, cum omnium idem finis esset» (Je me mis alors à penser, à part moi, combien il est absurde de craindre une chose plutôt qu'une autre, puisque toutes ont une même fin). Sénèque propose une réflexion morale et philosophique sur la sensibilité de l'homme aux maux qui l'assaillent. Ainsi, le seul contraste entre deux maux ou deux situations contraires peut suffire à l'ébranler, quelque prévenu qu'il soit contre les atteintes de la Fortune. Euvres de Sénèque le Philosophe avec la traduction française de la Collection Panckoucke, traduction de M. Charpentier-M. Lemaistre, t. I, Livre VI, lettre $n^{\circ}$ 57, Paris, Garnier, 1860. Une autre référence est envisageable: en effet, la citation apparaît également dans une lettre d'Erasme à Hector Boèce [1495]: «Itaque contentus mibi cantare et Musis, malui nibil edendo latere quam inepte scribendo imperitiam meam prodere» (Je me suis donc contenté de chanter pour moi-même et pour les Muses et j'ai préféré rester dans l'ombre en m'abstenant de publier plutôt que d'étaler mon insuffisance au grand jour). Ici encore, le contexte dans lequel Erasme emploie cette formule est fort différent de celui de la lettre de Voiture. Il est possible, toutefois, que celui-ci ait lu la lettre, et en garde le souvenir car Erasme y expose une conception de la poésie envisagée comme un divertissement que Voiture a pu reprendre à son compte à l'égard de sa production poétique et épistolaire. A Hector Boèce qui lui demande de lui montrer ses poésies, il répond qu'il s'est livré à la poésie dans sa jeunesse par jeu («et si quid puer aliquando lusi...»), mais qu'il se garde d'imiter ceux qui, par vanité, ne peuvent s'empêcher d'étaler leur insuffisance au grand jour et ne trouvent, pour les applaudir, que des admirateurs dignes de leur art. Il envoie néanmoins un ou deux poèmes à son correspondant par amitié pour lui, tout en lui recommandant instamment de ne laisser voir ces bagatelles à personne («caue illius nugas usquam efferas»). H.M. Allen, Opus epistolarum Erasmi Roterodami, t. I, Oxford, 1934, pour le texte latin. M. DelCOURT-Y. Rémy-A. Gerlo, La Correspondance d'Érasme, vol. I, University Press Bruxelles, 1979, pour la traduction en français.

(28) Ibid.

(29) Claude d'Urre du Puy-Saint-Martin de Chaudebonne (1584 ?-1644): premier maréchal des logis aux Suisses de la garde de Monsieur (1626), puis chancelier du duc d'Orléans.

(30) Le Comte-Duc d'Olivarès.

(31) Lettre à M. de Chaudebonne, Madrid, 9 juillet 1633, op. cit., t. I, p. 151. 
personnalité du destinataire qui était un homme dévot ${ }^{32}$. Cette façon d'aller délibérément à l'encontre des attentes du destinataire est manifeste dans une lettre écrite la même année où Voiture raille l'austérité de mœurs de Claude de Chaudebonne par une analogie entre le stoïcisme et la colique:

Que s'il est vrai qu'une des principales fins de la philosophie est le mépris de la vie, il n'y a point de si bon maître que la colique, et Socrate ni Platon ne persuadent pas si puissamment; elle m'a donné depuis une leçon de dix-sept jours dont il me souviendra longtemps, et m'a fait considérer beaucoup de fois combien nous sommes foibles, puisqu'il ne faut que trois grains de sable pour nous abattre. Que si elle me fait être de quelque secte, ce ne sera point de celle qui maintient que la douleur n'est point un mal, et que le sage est toujours heureux ${ }^{33}$.

La recherche de l'écart est encore plus accentuée, ici, et fait glisser le discours dans un registre burlesque puisque les références savantes à Socrate et Platon sont dénaturées par le registre «bas» de la colique. Il s'agit toutefois d'un burlesque adouci, «galant» ${ }^{34}$ car le langage lui-même n'est pas dégradé et le destinataire est l'objet d'une raillerie, non d'un sarcasme blessant. Cet usage tempéré du burlesque constitue une écriture codée, à lire au second degré qui, en transgressant apparemment le principe de la convenance, laisse apparaître en filigrane un entendement des références dégradées, une adhésion de l'épistolier à l'univers culturel du destinataire et enfin la littérarité du discours.

En se disant lui-même «négligent», Voiture prend donc une posture et offre, à ceux qui l'entendent ${ }^{35}$, une certaine visibilité aux principes qui règlent sa manière d'écrire. En donnant à ses lettres la forme d'une «conversation de personnes absen${\text { tes }\rangle^{36} \text {, il remet au goût du jour l'otium litteratum }}^{37}$ des Anciens et se conforme à un ethos galant qui consiste à ne pas faire montre de sa culture pour privilégier la sprezzatura caractérisant le Parfait courtisan ${ }^{38}$. C'est à ce titre que, dans la préface à la première édition de ses Euvres, il est présenté comme le modèle exemplaire du galant

(32) Claude de Chaudebonne se convertit en 1633, l'année où cette lettre est écrite. Il abandonne alors la vie mondaine et deviendra un membre ponctuel de la Compagnie du Saint-Sacrement.

(33) Lettre à M. de Chaudebonne, Madrid, février ou mars 1633 , ibid., t. I, p. 91 . On peut également se reporter au portrait burlesque que Voiture fait de Claude de Chaudebonne dans une lettre adressée la même année au cardinal de La Valette: lettre $n^{\circ} 67$, de Bruxelles, janvier ou février 1634, ibid., t. I, pp. 208-209.

(34) Le terme est employé par Claudine NéDÉLEC dans son étude sur le burlesque: Les Etats et empires du burlesque, Paris, Champion, «Lumière classique», 2004.

(35) On se réfère à Mme de Sévigné qui prend la défense de Voiture contre Méré, qui avait critiqué son style, en déclarant: «tant pis pour ceux qui ne l'entendent pas!», lettre du 24 novembre 1679 à Bussy-Rabutin, dans Correspondance, éd. R. DuCHÊNE, t. II, 712, p. 745. (Pour la critique faite par Méré, voir Discours de la justesse dans [Euvres, Les Conversations [1669], éd. Ch. BoudHors, Paris, 1930, t. I, pp. 95-112.)

(36) Madeleine DE Scudéry, De la manière d'écrire des lettres, dans De l'air galant et autres conversations, éd. D. DeNIS, Paris, Champion, 1998 («Sources classiques»), p. 154.

(37) Alain GÉNeTiot démontre comment une forme moderne de loisir mondain se développe dans le berceau de l'Hôtel de Rambouillet dans Poétique du loisir mondain de Voiture à La Fontaine, Paris, Champion, 1997. La liaison entre l'otium litteratum des Anciens et la nouvelle sociabilité mondaine est établie par Guez de Balzac dans quatre entretiens consacrés à l'urbanité des Romains et dédiés à la marquise de Rambouillet où il précise que l'oisiveté, pour être féconde, se goûte dans la conversation au sein d'une petite communauté choisie. (Le Romain, Suite d'un entretien de vive voix ou de la conversation des Romains, Mécénas, De la gloire [1644], éd. R. ZuBER, Paris, Champion, «Sources classiques», 1995). Voir Poétique..., pp. 431-506, et notamment, à propos de Guez de Balzac, pp. 431-440. Dès lors, la lettre, dont Guez de Balzac est l'un des premiers promoteurs, apparaît comme la forme écrite privilégiée de ce nouvel otium litteratum.

(38) BALDAZAR CASTIGlione: «...il faut fuir, autant qu'il est possible, comme un écueil très acéré et dangereux, l'affectation, et, pour employer peut-être un mot nouveau, faire preuve en toute chose d'une certaine désinvolture (sprezzatura), qui cache l'art et qui montre que ce que l'on a fait et dit est venu sans peine et presque sans y penser». Le Livre du Courtisan, éd. et trad. de A. Pons, I, XXVI, Paris, G. Lebovici, 1987 (rééd. Flammarion, «GF», 1991), p. 54. 
bomme $e^{39}$, car il incarne un comportement et une manière d'écrire qui revalorisent la négligence et le divertissement sans se confondre avec la frivolité et la superficialité que le terme «mondain» connote en français moderne.

En se démarquant des grands modèles épistolaires reconnus comme des textes littéraires, il ne se situe donc pas sur un plan différent, qui serait celui du discours familier, il rivalise plutôt avec eux. Aussi son écriture passe-t-elle également par une imitation des grands textes littéraires.

\section{Une «écriture du pastiche»}

La pratique de l'imitation est conventionnelle, au XVII ${ }^{\mathrm{e}}$ siècle, mais Voiture se distingue en puisant à des sources très diverses et en mêlant intiment des références et des procédures littéraires au discours épistolaire ancré sur le réel. Il s’inspire d'abord des vastes romans poétiques italiens ou espagnols, tels que Le Roland furieux de l'Arioste ou l'Amadis ${ }^{40}$, très en vogue dans les salons, car ils mêlent exploits chevaleresques et aventures amoureuses. Le pastiche d'œuvres parfaitement connues du lectorat mondain remplit alors une fonction sociale en établissant une connivence au sein du cercle des lecteurs. L'allusion au Roland furieux ou à l'Amadis s'impose naturellement dans les lettres qui accompagnent le présent d'un exemplaire de l'ouvrage. Comme dans la lettre à Mme de Saintot, celle que Voiture adresse au duc de Bellegarde pour accompagner l'Amadis joue d'une confusion délibérée entre le personnage et l'ouvrage:

Monseigneur, en une saison où l'histoire est si brouillée, j'ai cru que je vous pouvois envoyer des fables, et qu'en un lieu où vous ne songez qu'à vous délasser l'esprit, vous pourriez accorder à l'entretien d'Amadis quelques-unes de ces heures que vous donnez aux gentilshommes de votre province ${ }^{41}$.

De constantes analogies sont établies entre les personnages de la fiction et le destinataire de la lettre, indiquant que l'ouvrage a été choisi en fonction de la personnalité de ce dernier:

En effet, Monseigneur, vous avez fait voir à la France un Roger ${ }^{42}$ plus aimable et plus accompli que celui de Grèce et que celui de l'Arioste et, sans armes enchantées, sans le secours d'Alquife ni d'Urgande ${ }^{43}$, et sans autres charmes que ceux de votre personne, vous avez eu dans la guerre et dans l'amour les plus heureux succès qui s'y peuvent souhaiter. Aussi, à considérer cette courtoisie si exacte et qui ne s'est jamais démentie, cette grâce si charmante, dont vous gagnez les volontés de tous ceux qui vous voient, et cette grandeur et fermeté d'âme qui ne vous a jamais permis d'aller contre le devoir, ni même contre la bienséance, il est bien difficile de ne se pas imaginer que vous êtes de la race des Amadis ${ }^{44}$.

(39) «Elles [Les dames] ont jugé qu'il approchait de fort près des perfections qu'elles se sont proposées pour former celui que les Italiens nous décrivent sous le nom du parfait courtisan, et que les Français appellent un galant homme». MARTIN PinChesne, dans Euvres de M. de Voiture, op. cit., t. I, p. 8 .

(40) Roman de chevalerie espagnol écrit et publié en 1508 par Garci Rodriguez de Montalvo. Il est traduit en français par Nicolas de Herbelay des Essarts et publié par Jean Janot, Vincent Sertenas et Longis à Paris en 1540. 1er livre, publié sur l'éd. originale par Hughes Vaganay, nouvelle éd. avec introduction, glossaire et relevé de variantes établie par Yves GIRAud, t. I, chap. I-XXI, Société des Textes Français Modernes, Paris, Nizet, 1986.

(41) La lettre est adressée au duc de Bellegarde ( ?-1646) après qu'il ait été exilé en Bourgogne, dont il était gouverneur, sur ordre du cardinal de Richelieu. Lettre $n^{\circ}$ 5, 1627, op. cit., t. I, pp. 31-33.

(42) Le duc de Bellegarde se nommait Roger de Saint-Lary.

(43) Allusion à un passage d'Amadis de Gaule.

(44) Ibid. 
Dans un univers de l'entre-deux mêlant individus réels et personnages, la lettre semble brouiller les frontières entre les types de discours, mais le pastiche souligne la littérarité du discours par la proximité qu'il établit avec le texte littéraire. Cette littérarité s'affiche presque concrètement dans la lettre à Mme de Saintot car Voiture l'a fait imprimer en tête de l'exemplaire du Roland furieux qu'il offrait à la dame. Le discours épistolaire échappe ainsi à la matérialité de la lettre et pénètre dans l'espace de l'objet littéraire. Un processus similaire apparaît à l'intérieur même du discours, dans une lettre au cardinal de La Valette. Voiture évoque les «exploit(s)» de la campagne militaire menée par le cardinal en le comparant aux personnages de l'Arioste, et représente son parcours comme les chapitres d'un livre inachevé:

Monseigneur, si vous vous souvenez de la passion que vous m'avez vue autrefois pour Renaud et pour Roger, vous ne douterez pas de celle que j'ai à cette heure pour ce qui vous regarde, puisque vous faites en pourpoint tout ce que ceux-là faisaient avec des armes enchantées. [...] Mais quand je songe [...] que je ne suis pas assuré de ce qui arrivera à la fin du livre, je ne saurais jouir qu'avec inquiétude de la gloire que tout le monde vous donne, et la crainte de l'avenir ne me laisse pas bien sentir le contentement des choses présentes ${ }^{45}$.

Le «livre», ici, n'a pas de matérialité, mais il est constitué par les lettres de Voiture qui accompagnent le cardinal en célébrant ses victoires et en le consolant de ses revers.

La pratique du pastiche se fait plus précise et plus subtile dans les célèbres lettres en vieux langage. Voiture associe des allusions à diverses œuvres, chansons de geste, romans courtois médiévaux et romans d'aventure contemporains ${ }^{46}$, imite à la fois le style et la langue des romans médiévaux, et transfigure les éléments du réel. Ainsi, le siège de $\mathrm{Cazal}^{47}$, fait par les armées alliées du roi d'Espagne et du duc de Savoie, est-il transformé en histoire de rapt et la ville est représentée sous les traits:

[d'] une damoiselle qui Cazalie est nommée, moult prisée et cherie de ceux du païs, et de maints grands seigneurs d'étranges terres desirée, comme celle qui est de moult beau viaire et bien addressée de tous ses membres, avenante et de si plaisant regard, que c'est un déduit à regarder ${ }^{48}$.

L'actualité politique fait l'objet d'une réécriture sur un mode romanesque, et les acteurs de l'Histoire sont assimilés à des personnages romanesques selon un procédé relevant davantage de la métaphore que de la comparaison: le prince Thomas de Savoie est «un geant dépiteux et felon» à la solde de «son seigneur le soudan des Iberiens» qui «convoitoit [la damoiselle] pour la mettre en servage et lui tollir son bonneur». Mais celle-ci est sous la protection de «l'invincible chevalier qui porte d'azur à

(45) Lettre au cardinal de La Valette, Mars 1639, ibid., t. I, pp. 322-323.

(46) Voiture imite la langue et le style des romans du Moyen Âge sans faire de référence précise à l'un d'entre eux en particulier. Le comte de Saint-Aignan, qui était à l'origine de cet échange de lettres en «vieux langage», fait allusion à La jeune Alcidiane de Gomberville en signant: «Dom Guilan le Pensif, sire de l'Isle Invisible».

(47) En italien, Casale Monferrato, ancienne capitale du Montferrat, en Italie septentrionale. La ville, qui appartenait aux Gonzague, ducs de Mantoue et marquis de Montferrat, protégés du roi de France, est d'abord convoitée par le duc de Savoie, possesseur du Piémont, puis par le roi d'Espagne, maître du Milanais. En 1629-1630, Charles-Emmanuel de Savoie s'allie au roi d'Espagne pour le partage du Montferrat dont ils contestent la propriété à Charles de Gonzague-Clèves, duc de Nevers et de Rethel, né sujet français. Les deux armées alliées font le siège de Cazal, défendue par une armée française dirigée par le comte d'Harcourt. La paix sera négociée par Mazarin alors au commencement de sa carrière.

(48) Ce passage et les suivants sont extraits de la lettre aux comtes de Guiche et de Saint-Aignan et à I. Arnauld de Corbeville, 1640, ibid., t. II, p. 265-266. 
trois fleurs d'or» et du «chevalier faé aux vermeilles connoissances» qui, à l'inverse du Soudan, «aime la pucelle par bonne amour, et sans vilenie, seulement pour la franchise d'elle garder». Ces derniers dépêchent «Harcuriel des Isles périlleuses» qui, après une «mout caveuse et cruelle bataille» se rend maître des «gloutons», du géant et «toute sa mesgnie fit mise à occision». La lettre apparaît moins comme un discours épistolaire égayé par des références à des ouvrages littéraires que comme une véritable fiction littéraire et linguistique ${ }^{49}$ tirant sa saveur de ses affinités avec le monde du réel. D'ailleurs, ces lettres, échangées d'abord dans le cercle étroit de quelques correspondants, font rapidement l'objet d'une large diffusion dans le milieu mondain qui consacre explicitement leur statut littéraire.

Enfin, Voiture s'inspire également du style d'auteurs qui n'étaient pas particulièrement en vogue dans son milieu, comme Clément Marot à qui il rend de nombreux hommages dans ses poésies. Le pastiche ne s'impose plus, alors, pour établir une connivence avec le destinataire, mais comme ferment d'un renouvellement des modèles alors en usage. Dans des lettres majoritairement vouées à l'expression de l'éloge et du compliment, le style du badinage marotique contraste avec les modalités traditionnelles du registre encomiastique. La lettre de la Carpe au Brochet, par exemple, adressée au duc d'Enghien pour le féliciter d'avoir fait passer le Rhin à son armée, prend la forme d'une sorte de fable qui peut rappeler la célèbre épître de Marot à son ami Lyon Jamet ${ }^{50}$. Pour évoquer la traversée du fleuve, Voiture transpose les individus et la campagne militaire dans l'univers des poissons. Toutefois, alors que dans son épî́tre à Lyon Jamet Marot s'adressait à un ami, Voiture enfreint les codes de l'échange avec les Grands supposant l'emploi d'un registre élevé en se situant dans le registre d'un burlesque «plaisant»: il dégrade l'image du prince du sang en l'assimilant à un brochet et l'interpelle familièrement par l'apostrophe: «Eh! bonjour, mon compère le Brochet!». Pour sa part, il signe ironiquement «La Carpe», endossant l'identité d'un poisson qui symbolise le laconisme, après s'être montré fort disert. Le discours séduit le destinataire non par l'élévation du style, mais par sa fantaisie car la fable est prétexte à de multiples jeux sur le langage. Voiture remotive le sens d'expressions imagées passées dans le vocabulaire courant qui exploitent le vocabulaire de la mer et des poissons: «..connoissant votre force et combien vous aimez à nager en grande eau ${ }^{51}$, j'avois bien cru que [les eaux du Rhin] ne vous feroient point de peur...»; «Quoique vous ayez été excellent jusqu'ici à toutes les sauces où l'on vous a mis, il faut avouer que la sauce d'Allemagne vous donne un grand goût...»; «Il n'y a point d'eau si trouble, si creuse ni si rapide où vous ne vous jetiez à corps perdu...»; «[Un vieux saumon] nous dit [...] que les baleines de la mer Atlantique suoient à grosse goutte dès qu'elles vous

(49) A propos de l'imitation de la langue des romans médiévaux, JEAN FRAPPIER précise qu'il entre dans le langage employé par Voiture à la fois une part de création et une part de fidélité à un langage authentique: «On perdrait son temps à vouloir la juger [son amusette archaïsante] avec une rigueur philologique. Le pasticheur s'est octroyé des licences ou une part de création personnelle; mais, dans l'ensemble, son 'vieux langage' n'est pas de fantaisie; c'est du moyen français, celui qu'il lisait dans les versions des 'vieux romans' imprimées dans la première moitié du XVI ${ }^{\text {e }}$ siècle; elles gardaient évidemment des tours et des vocables déjà anciens à cette époque. Pour la syntaxe, Voiture ne s'entend pas trop mal à faire à bon escient les omissions du pronom sujet ainsi que les inversions. Son vocabulaire mérite plus d'attention, et il serait sans doute instructif d'en dresser un glossaire complet
[...]. Enfin, il a bien attrapé le rythme un peu lent et flottant des récits chevaleresques en prose $[\ldots]$.. «Voiture amateur de vieux romans», dans Amour courtois et Table ronde, Genève, Droz, «Publications romanes et françaises», 1973, p. 294.

(50) Marot pastiche la fable d'Esope Le lion et la rat en se fondant d'une part sur un jeu sur le nom «Lyon» de son ami, et d'autre part sur les circonstances de la rédaction de l'épître: grâce, sans doute, à l'intervention de ce dernier, il vient de sortir de la prison où il avait été enfermé sur accusation de luthéranisme et transféré dans une prison plus acceptable. Voir L'Adolescence clémentine, éd. G. DefauX, «Classiques Garnier», Paris, Dunod, 1996, pp. 92-94 pour l'épître et pp. 476-477 pour l'élucidation du contexte.

(51) Nous soulignons cette expression et les suivantes. 
entendoient seulement nommer» $>^{52}$, etc. De même, se fondant sur la double référence au monde fictif des poissons et à l'univers réel de la guerre de Trente Ans; il emploie certains mots avec un sens littéral et un sens figuratif, comme les «lauriers» qui «relèvent merveilleusements ${ }^{53}$ le Brochet. Cette manière plaisante d'utiliser la catachrèse et la syllepse souligne la part d'invention qui entre dans cette nouvelle réécriture de l'actualité politique.

L'imitation, chez Voiture, se distingue donc par une association de modèles d'inspiration différents. Les lettres adressées à Mlle Paulet depuis Ceuta sont demeurées célèbres parce qu'elles se fondent sur l'alliance insolite du pastiche des $M e ́-$ tamorphoses d'Ovide et du style «naïf» de la fable avec les topoï amoureux issus de la poésie courtoise qui ont été récupérés par les Pétrarquistes. La référence à la fable est appelée par le surnom «La Lionne» donné dans le cercle de l'Hôtel de Rambouillet à la destinataire pour sa chevelure abondante. Profitant de son incursion en Afrique, où vivent de véritables lions, Voiture représente Mlle Paulet comme une lionne qui aurait pris l'apparence d'une femme. Dans la première lettre, il déclare qu'il ira voir ses «parents» pour la demander en mariage et, après avoir ménagé un moment de suspense, ajoute:

A ce que j'entends, ce sont gens peu accostables. J'aurai de la peine à les trouver. On dit qu'ils doivent être au fond de la Libye, et que les lions de cette côte sont moins nobles et moins grands ${ }^{54}$.

La deuxième lettre envoyée de Ceuta à la dame accompagne le présent de lions de cire rouge que Voiture traite comme des personnages de fable: ils sont représentés à la fois comme des lions véritables et comme des nobles contraints à l'exil. Mais la transposition du discours dans un univers fabuleux et le thème de la métamorphose servent surtout de fondement à un détournement ironique des topö̈ amoureux car l'image de la lionne est associée à l'isotopie de la cruauté féminine:

J'envoie dès demain des cartels aux Mores de Maroc et de Fez, où je m'offre à soutenir que l'Afrique n'a jamais rien produit de plus rare et de plus cruel que vous ${ }^{55}$.

Voiture superpose l'image de la lionne et celle de la Belle Dame sans mercy, employant l'adjectif «cruel» à la fois au sens métaphorique et littéral. L'humour de la seconde lettre se fonde sur une valorisation ironique du lexique de la cruauté qui, en tant que ricor amoureuse, projette l'image d'une femme exigeante et difficile à séduire, mais renvoie, dans la lettre, à l'appétit des bêtes fauves:

Je vous assure, mademoiselle qu'ils [ces lions] sont estimés les plus cruels et les plus sauvages de tout le pays, et j'espère que vous en aurez toute sorte de contentement. Il y a avec eux quelques lionceaux qui, pour leur jeunesse, n'ont encore pu étrangler que des enfants et des moutons; mais je crois qu'avec le temps ils seront gens de bien, et qu'ils pourront atteindre à la vertu de leurs pères. Au moins sais-je bien qu'ils ne verront rien auprès de vous qui leur puisse radoucir ou rabaisser le cœur et qu'ils y seront aussi bien nourris que s'ils étaient dans leur plus sombre forêt d'Afrique ${ }^{56}$.

(52) Lettre $n^{\circ} 155$ au duc d'Enghien, novembre 1643 , ibid., t. I, pp.402-403.

(53) Ibid., t. I, p. 402.

(54) Lettre $n^{\circ} 53$, à Mlle Paulet, ibid., t. I, pp.
162-163.

(55) Ibid., t. I, p. 162. 168. 
L'imitation très libre des Métamorphoses et du badinage marotique subvertit entièrement les images traditionnelles de l'amour léguées par la Courtoisie ou le Pétrarquisme. Leur sens est affadi mais elles héritent d'une tonalité enjouée qu'elles ne possédaient pas originellement. Elles n'expriment plus l'intensité du sentiment amoureux comme dans la poésie du Moyen Age ou du Xvi ${ }^{\mathrm{e}}$ siècle, mais une forme hyperbolique et plaisante du compliment adressé aux dames.

Le recours régulier au pastiche met donc en évidence la littérarité des lettres de Voiture. En mêlant des sources d'inspiration diverses et en produisant des effets de décalage par rapport à ses modèles ou aux circonstances de l'échange épistolaire, il renouvelle à la fois l'expression de l'éloge et le champ de l'écriture épistolaire. Il assure en effet la promotion d'un nouveau modèle épistolaire nommé, à cette époque, «lettre galante» ${ }^{57}$. Elle se caractérise par une distance de badinage qui lui donne un aspect futile et artificiel si on l'envisage comme un discours familier, mais révèle, si on la considère comme un texte littéraire, une pratique raffinée et inventive de l'imitation.

$$
* * *
$$

L'originalité des lettres de Voiture réside donc dans cette «écriture du pastiche» qui met en application l'imitation telle qu'elle était conçue au XVII siècle. Il s'agit surtout de s'approprier les thématiques et les procédures stylistiques empruntées pour alimenter un véritable travail de création ${ }^{58}$. A cela s'ajoute, chez Voiture, la gourmandise d'un grand lecteur qui privilégie un principe de plaisir, pastiche les œuvres qui lui plaisent, assimilant d'autant plus intimement leurs caractéristiques pour développer une manière qui lui est propre.

Bien que ses lettres aient fait l'objet d'une minoration à partir du XVII siècle pour des raisons diverses ${ }^{59}$, force est donc de constater qu'elles occupent une position

(57) C'est l'expression couramment employée à propos des lettres de Voiture. On la trouve notamment chez Vaugelas (Remarques..., «galant, galamment», op. cit.) et Richelet (Dictionnaire françois..., op. cit., article «galant»). Après la publication des Euvres de Voiture, cette appellation entre dans le vocabulaire littéraire et dans le vocabulaire courant pour désigner le type de lettres qu'il a mis en vogue. Madeleine de Scudéry consacre un développement particulier à la lettre galante dans un texte théorique présenté sous forme de «Conversation»: De la manière d'écrire des lettres dans De l'air galant et autres conversations, op. cit.

(58) Le Père Bouhours fait l'éloge de cette imitation créative en reprenant le topos des abeilles qui puisent dans les fleurs une matière qu'elles assimilent pour en faire leur miel, et présente cette pratique de l'imitation comme la marque de distinction du Bel esprit, contrairement à ceux qui «font de l'esprit» en cherchant à se faire valoir par de «faux brillants». Voiture, à ses yeux, incarne cet idéal de façon exemplaire: «Je veux bien aussi qu'il [le bel esprit] se serve dans les rencontres des pensées des bons auteurs, pourvu qu'il y ajoute des beautés nouvelles; et qu'à l'exemple des abeilles, qui changent en miel ce qu'elles prennent des fleurs, non seulement qu'il choisisse ce qu'il y a de bon dans les livres, mais encore qu'il se fasse propre ce qu'il choisit, et qu'il le rende meilleur par l'usage qu'il en fait. C'est un des grands talents de Voiture: en imitant les autres, il s'est rendu inimitable: il savait admirablement l'art de mettre en œuvre, et de faire valoir les pensées des auteurs; les traits qu'il emprunte quelquefois de Térence et d'Horace, semblent faits pour son sujet, et sont bien plus beaux dans les endroits où il les met, que dans ceux d'où il les a pris: de même que les pierres précieuses sont plus belles dans les bagues où on les enchâsse, que les roches d'où on les tire». Entretiens d'Ariste et d'Eugène, IV: «Le Bel esprit», éd. F. BRunOt, Paris, Armand Colin, 1962, p. 119-121. Comme le souligne Alain Génetiot, cette manière d'imiter des sources diverses en développant un style original sera aussi ce qui distinguera la production poétique de La Fontaine. Poétique du Loisir mondain..., op. cit., «La théorie de l'imitation-émulation», pp. 27-37.

(59) Un courant de réaction contre la littérature galante dans son ensemble se fait jour à partir du $\mathrm{XVIII}^{\mathrm{e}}$ siècle. Voltaire, notamment, dans son effort pour faire du siècle de Louis XIV un équivalent moderne du siècle d'Auguste, porte des jugements sévères sur les poètes mondains du temps de Louis XIII ou de la Régence de Marie de Médicis (VoLTAIRE, Le Temple du goût, dans Euvres complètes, t. XII, Paris, Baudoin, 1829). Après lui, la critique du XIX ${ }^{\mathrm{e}}$ siècle assimile le courant littéraire de la galanterie au phénomène social et anecdotique de la préciosité. Pour que soit de nouveau reconnue la nature littéraire de la galanterie et, avec elle, 
charnière dans l'Histoire littéraire. Elles jouent un rôle fondateur dans la formation de l'épistolaire en tant que genre littéraire et assurent la reconnaissance de la lettre galante ou mondaine qui fera flores par la suite, comme en témoignent les lettres de Mme de Sévigné. Peut-être sont-elles aussi à l'origine d'un intérêt particulier, à cette époque, pour la lettre privée dont témoigne l'engouement pour les Lettres portugaises, à la fin du siècle. Enfin, en prenant la forme du discours familier, elles ont contribué à promouvoir un idéal du naturel qui, comme on vient de le voir, suppose en réalité une grande virtuosité littéraire pour dissimuler l'artifice et produire une image plus belle que la nature elle-même. Voiture se trouve ainsi à l'origine du développement de l'esthétique galante qui atteint une pleine visibilité à partir de la publication de ses Euvres et devient par la suite le mode d'expression et de reconnaissance des Modernes.

SOPHIE ROLLIN

de la lettre familière et d'auteurs tels que Voiture, il faudra attendre un nouveau courant de recherches inauguré notamment par Alain Viala dans les années 1990. Voir à ce sujet AlAIN GÈNETIOT, Des Hommes illustres exclus du Pantbéon: les poètes mondains et galants, dans «Littératures classiques» n 19 , automne 1993 , pp. 215-235 et Alain VIALA, Qui t'a fait Minor? Galanterie et classicisme, dans «Littératures classiques» $\mathrm{n}^{\circ} 31$ : Les «Minores», automne 1997, pp. 115-134. 